\title{
Performance Study of a Class of Irregular Near Capacity Achieving LDPC Codes
}

\author{
Francesca Vatta, Alessandro Soranzo, Massimiliano Comisso, Giulia Buttazzoni, and Fulvio Babich
}

\begin{abstract}
This paper investigates the performance of a class of irregular low-density parity-check (LDPC) codes through a recently published low complexity upper bound on their beliefpropagation decoding thresholds. Moreover, their performance analysis is carried out through a recently published algorithmic method, presented in Babich et al. 2017 paper. In particular, the class considered is characterized by variable node degree distributions $\lambda(x)$ of minimum degree $i_{1}>2$ : being, in this case, $\lambda^{\prime}(0)=\lambda_{2}=0$, this is useful to design LDPC codes presenting a linear minimum distance growth with the block length with probability 1, as shown in Di et al.'s 2006 paper. These codes unfortunately cannot reach capacity under iterative decoding, since the achievement of capacity requires $\lambda_{2} \neq 0$. However, in this latter case, the block error probability might converge to a constant, as shown in the aforementioned paper.
\end{abstract}

\section{INTRODUCTION}

LDPC codes are a class of channel block codes, first introduced in the 1960's by Robert Gallager [1], representing the leading edge in modern channel coding. Due to the technical limitations of that age, LDPC codes were scarcely considered for almost 30 years, apart from Tanner's generalized LDPC definition and graphical representation, presented in his 1981 paper [2] (which was later called Tanner graph), and were reinvented in the mid 1990's by MacKay [3] and Luby et al. [4]. After being included in modern communication standards such as digital video broadcasting DVB-S2 (satellite communication), ITU-T G.hn (home networking), and DOCSIS 3.1 (cable) standards, they are also used in the IEEE802.11 (Wi-Fi) [5], [6], 802.16e (Wi-MAX), and 10G-BaseT Ethernet standards, and have been also proposed as component codes of product code structures [7] for the next generation digital terrestrial broadcasting transmission system [8]. Moreover, they were recently adopted, together with polar codes, by the fifth-generation $(5 \mathrm{G})$ new radio $(\mathrm{NR})$ standard (see, e.g., [9] [11]).

As first noticed by Gallager in his aforementioned introductory work, limited to regular LDPC codes, these exhibit the

Manuscript received December 30, 2020; revised March 22, 2021. Date of publication March 30, 2021. Date of current version March 30, 2021.

F. Vatta, M. Comisso, G. Buttazzoni, and F. Babich are with the DIA, University of Trieste, Trieste, Italy. A. Soranzo is with the DMG, University of Trieste, Trieste, Italy (e-mails: vatta@units.it, soranzo@units.it, mcomisso@units.it. gbuttazzoni@units.it, babich@units.it).

Part of this work was presented at the International Conference on Software, Telecommunications and Computer Networks, SoftCOM '20, Hvar, Croatia, September 17-19, 2020.

This work has been partially supported by the Italian Ministry of University and Research (MIUR) within the project FRA 2018 (University of Trieste, Italy), entitled "Integrazione cUBEsat-Reti 5G: analisi del livello di accesso e sviluppo di sistemi d'antenna".

Digital Object Identifier (DOI): 10.24138/jcomss-2020-0009 so called "threshold phenomenon". Namely, an upper bound for the channel noise can be defined by the noise threshold so that, if the channel noise is maintained below this threshold, the probability of lost information can be made as small as desired. Later, Luby et al. showed that irregular LDPC codes perform better than regular ones [4], and exhibit the threshold phenomenon, too. In this work, they also showed that their hard-decision decoding process may be analyzed considering an individual edge between a variable node $m$ and a check node $c$ and an associated tree, rooted in $m$, describing its neighborhood, as shown in Fig. 1 of [4]. Denote with $p_{l}$ the probability that $m$ sends $c$ an incorrect value at the $l$-th iteration. Following the work of Gallager [1], in [4] a recursive equation describing the evolution of $p_{l}$ was determined over a constant number of iterations.

LDPC codes are capacity-approaching codes, which means that practical constructions exist that allow the noise threshold to be set very close to the theoretical maximum (the Shannon limit) for a symmetric memoryless channel. Thus, the problem of an easy evaluation of the threshold, and, in general, of the performance of belief propagation decoding is important to allow the design of capacity-approaching codes, based on noise threshold maximization (see, e.g., [12] where, using the analysis outlined in [13], a custom software based on [14] was employed to simulate the performances of punctured LDPC codes over an additive white Gaussian noise (AWGN) channel, assuming a binary phase shift keying modulator).

Our paper [15] was focused on the investigation about the usefulness of a low complexity upper bound on beliefpropagation decoding thresholds, recently published in [16], for the class of irregular LDPC codes characterized by variable node degree distributions

$$
\lambda(x)=\sum_{i=i_{1}}^{d_{l}} \lambda_{i} x^{i-1}
$$

of minimum degree $i_{1}>2$, being $\lambda_{i}$ the fraction of edges in the Tanner graph connecting to a degree- $i$ variable node and $d_{l}$ the maximum variable node degree. This investigation was absolutely novel. In fact, the above mentioned upper bound of $[16]^{1}$ was conceived as an algebraic method to calculate

\footnotetext{
${ }^{1}$ In [16], three low complexity upper bounds to the exact belief-propagation decoding thresholds were derived, by applying the result of [20] to the asymptotical behavior of $\Delta(s, t)$, defining the difference between the mean values of a generic check node output messages at the $l$-th and at the $(l-1)$-th iteration. As shown in [16], the second and third bounds, therein presented, called $s_{\text {approx }}^{*}$ and $s_{\text {Jensen, }}^{*}$, respectively, are applicable only to a class of LDPC codes, namely, to the class of codes presenting variable node degree distributions $\lambda(x)$ of minimum degree $i_{1}=2$. The first bound found in [16] instead, called $s_{\text {bound }}^{*}$, is more generically valid $\forall i_{1} \geq 2$.
} 
upper bounds on belief-propagation decoding thresholds for the class of irregular LDPC codes characterized by $i_{1} \geq 2$, since, in the literature (see, e.g., [17] and [18]), only the case with $i_{1}=2$ was considered before, as far as the upper bounds on thresholds and on the coefficient $\lambda_{i_{1}}$ (stability condition) are concerned (see Example 12 of $[18]^{2}$ ).

In the present paper, in addition to what already presented in [15], we investigate the suitability of a recently published algorithmic method, presented in $[20]^{3}$, to find the convergence conditions of the above mentioned recursive sequence describing the evolution of $p_{l}$ in [4]. This investigation is motivated by the need of analyzing the class of irregular near capacity achieving LDPC codes therein considered, characterized by variable node degree distributions $\lambda(x)$ of minimum degree $i_{1}>2$. This analysis is completely novel since, so far, the performance analysis we conducted in [21] and [22] was focused on the class of LDPC codes presenting degree distributions $\lambda(x)$ of minimum degree $i_{1}=2$. In order to accomplish this task, we investigated on the role played by the product $\lambda^{\prime}(0) \rho^{\prime}(1)$ in determining this performance, being $\rho(x)=\sum_{j=2}^{d_{r}} \rho_{j} x^{j-1}, \rho_{j}$ the fraction of edges connecting to a degree- $j$ check node, and $d_{r}$ the maximum check node degree. However, as shown in [21], being $\lambda^{\prime}(0)=\lambda_{2}$, this analysis is feasible only for the class of LDPC codes presenting $\lambda_{2} \neq 0$.

The investigation on the class of irregular LDPC codes characterized by variable node degree distributions $\lambda(x)$ of minimum degree $i_{1}>2$ is interesting because the fact that, in this case, $\lambda^{\prime}(0)=\lambda_{2}=0$, implies that these codes present a linear minimum distance growth with the block length with probability 1 , as shown in [23]. These codes unfortunately cannot reach capacity under iterative decoding, since the achievement of capacity requires $\lambda_{2} \neq 0$. However, in this latter case, the block error probability might converge to a constant [23], since, as shown in [24], in order to have zero word error probability, it is necessary to have $\lambda_{2}=0$. This was proved in [24] by the following argument: if $\lambda_{2}>0$, then in the ensemble, as the block length $n \rightarrow \infty$, the average number of weight 2 codewords is bounded away from zero. Hence even a maximum likelihood decoder would have nonzero decoding error probability, fact that does not happen with $\lambda_{2}=0$.

The paper is organized as follows. In Section II we recall the first upper bound to the belief propagation decoding thresholds already derived in [16], since the paper is focused on it. In Section III, the method outlined in [4] for designing irregular graphs is recalled, based on a recursive equation describing the evolution of the above mentioned $p_{l}$, for a given value of the parameter $p_{0}$. This is necessary so as to provide the reader with a reference to the mathematical functions needed in the following. In Section IV we rewrite the mathematical method presented in [20] in a form suitable to obtain the

\footnotetext{
${ }^{2}$ As far as the LDPC codes found in [18] are concerned, all presenting variable node degree distributions $\lambda(x)$ of minimum degree $i_{1}=2$, in [16] and [19] were presented the upper bounds to their exact belief-propagation decoding thresholds.

${ }^{3}$ The method consists in solving a problem of quadratic degeneracy instead of looking for the conditions guaranteeing the convergence of a certain sequence, task which normally has to be performed manually, i.e., by repeated trials.
}

"supremum $p^{*}$ of all values of $p_{0}$ for which the sequence $p_{l}$ is monotonically decreasing and hence converges to 0" [4]. In Section $\mathrm{V}$ some numerical results are given to investigate the usefulness of the above mentioned bound in determining the performance of an irregular LDPC code characterized by variable node degree distributions $\lambda(x)$ of minimum degree $i_{1}>2$. Moreover, some numerical results are also given in terms of the obtainable $p^{*}$ values for the class of irregular LDPC codes considered in [4] reporting some simulation results, too. Finally, Section VI summarizes the results of the paper.

\section{UPPER BOUNDS ON LDPC CODES DECODING THRESHOLDS}

To determine the upper bounds on thresholds, first of all we have to determine the asymptotical behaviour of (18) in [15] for $t \rightarrow \infty$. The first upper bound, called $s_{\text {bound }}^{*}$ in [16], was found computing $\phi(x)$, defined in (9) of [15], with $x \geq 10$. To this end, we have added a further invertible approximation of the function $\phi(x)$, the derivation of which is given in the Appendix of [16]. To obtain the second upper bound, called $s_{\text {approx }}^{*}$ in [16], we have used the approximation (16) of [16] (that was implicitly used in [17]). The third upper bound, called $s_{\text {Jensen }}^{*}$ in [16], was obtained applying the Jensen's inequality to the latter asymptotical approximation.

\section{A. Upper bound on LDPC codes decoding thresholds holding for $i_{1} \geq 2$}

As far as the above mentioned first upper bound on LDPC codes thresholds (called $s_{\text {bound }}^{*}$ in [16]) is concerned, given a degree distribution $\lambda(x)$ of minimum degree $i_{1} \geq 2$, defining $z(s, t):=\frac{s+\left(i_{1}-1\right) t}{2}$ and $A_{j}:=\frac{1}{(j-1)^{2} \lambda_{i_{1}}^{2}}$, the following lemma is proved in [26].

Lemma: As $t \rightarrow \infty$, being $W(\cdot)$ the Lambert- $W$ function:

$$
f(s, t)=2 \sum_{j=2}^{d_{r}} \rho_{j} W\left(A_{j} z(s, t) \mathrm{e}^{z(s, t)}\right)+O\left(t^{-1}\right)
$$

With this $f(s, t)$ and remembering that $\frac{\mathrm{d} W(x)}{\mathrm{d} x}=\frac{1}{x+e^{\mathrm{W}(x)}}$ :

$f_{t}(s, t)=2 \sum_{j=2}^{d_{r}} \rho_{j} \frac{z_{t}(s, t) e^{z(s, t)}(1+z(s, t))}{z(s, t) e^{z(s, t)}+e^{\mathrm{W}\left(A_{j} z(s, t) e^{z(s, t)}\right)-\log A_{j}}}$

Applying (15) of [15] to (1) and (2) we get:

$$
\left\{\begin{array}{l}
2 \sum_{j=2}^{d_{r}} \rho_{j} \mathrm{~W}\left(A_{j} z(s, t) e^{z(s, t)}\right)=t \\
2 \sum_{j=2}^{d_{r}} \rho_{j} \frac{z_{t}(s, t) e^{z(s, t)}(1+z(s, t))}{z(s, t) e^{z(s, t)}+e^{\mathrm{W}\left(A_{j} z(s, t) e^{z(s, t)}\right)-\log A_{j}}}=1
\end{array}\right.
$$

and (18) of [15] can be rewritten as:

$$
\left\{\begin{array}{l}
2 \sum_{j=2}^{d_{r}} \rho_{j} \mathrm{~W}\left(A_{j} z(s, t) e^{z(s, t)}\right)-t=0 \\
2 \sum_{j=2}^{d_{r}} \rho_{j} \frac{z_{t}(s, t) e^{z(s, t)}(1+z(s, t))}{z(s, t) e^{z(s, t)}+e^{\mathrm{W}\left(A_{j} z(s, t) e^{z(s, t)}\right)-\log A_{j}}}-1=0
\end{array}\right.
$$

Its solution ( $s_{\text {bound }}^{*}, t_{\text {bound }}^{*}$ ), obtained applying the instruction set produced in [20], determines the bound $\sigma_{\text {bound }}^{*}=$ $\sqrt{\frac{2}{s_{\text {bound }}^{*}}}$, which is valid $\forall i_{1}$, unlike the other two $\left(s_{\text {approx }}^{*}\right.$ and $s_{\text {Jensen }}^{*}$ ) reported in [16], which hold both for $i_{1}=2$ only. 


\section{IRREGULAR GRAPHS DESIGN}

Irregular LDPC codes [18] are defined by specifying the distribution of the node degrees in their Tanner graphs. In particular, in the edge-perspective degree distribution, $\lambda_{i}$ is the fraction of edges in the Tanner graph connecting to a degree- $i$ variable node, and $\rho_{j}$ is the fraction of edges connecting to a degree- $j$ check node.

Consider an irregular LDPC code with edge-perspective degree distributions $\lambda(x)$ and $\rho(x)$, defined as

$$
\begin{aligned}
\lambda(x) & :=\sum_{i=i_{1}}^{d_{l}} \lambda_{i} x^{i-1} \\
\rho(x) & :=\sum_{j=2}^{d_{r}} \rho_{j} x^{j-1}
\end{aligned}
$$

being $d_{l}$ (respectively $d_{r}$ ) the maximum variable (respectively check) node degree. The $d_{l}$-tuple $\left\{\lambda_{i}\right\}$ and $d_{r}$-tuple $\left\{\rho_{j}\right\}$ both add up to 1 .

Gallager's hard-decision decoding approach [1] has been generalized in [4] to the case of irregular graphs, in order to consider the varying degrees of the variable and check nodes. It may be analyzed considering an individual edge between a variable node $m$ and a check node $c$ and an associated tree, rooted in $m$, describing its neighborhood, as shown in Fig. 1 of [4]. Denote with $p_{l}$ the probability that $m$ sends $c$ an incorrect value at the $l$-th iteration. Following the work of Gallager, in [4] is determined a recursive equation describing the evolution of $p_{l}$ over a constant number of iterations of the message passing decoding algorithm used for hard-decision decoding. Given an irregular LDPC code with given distributions (5) and (6), and fixing a $p_{0}$ value, this recursive equation is simply given by

$$
p_{l}=f\left(p_{0}, p_{l-1}\right)
$$

where, for $0 \leq p_{0} \leq 1$ and $0 \leq p \leq 1$, the function $f\left(p_{0}, p\right)$ is defined as

$$
f\left(p_{0}, p\right):=p_{0}-\sum_{i=i_{1}}^{d_{l}} \lambda_{i} f_{i}\left(p_{0}, p\right)
$$

through $f_{i}\left(p_{0}, p\right)$, defined as

$$
\begin{aligned}
f_{i}\left(p_{0}, p\right):= & p_{0} \sum_{k=b_{i}\left(p_{0}, p\right)}^{i-1}\left(\begin{array}{c}
i-1 \\
k
\end{array}\right)\left[\frac{1+\rho(1-2 p)}{2}\right]^{k} \\
& \cdot\left[\frac{1-\rho(1-2 p)}{2}\right]^{i-1-k} \\
& +\left(1-p_{0}\right) \sum_{k=b_{i}(p)}^{i-1}\left(\begin{array}{c}
i-1 \\
k
\end{array}\right)\left[\frac{1-\rho(1-2 p)}{2}\right]^{k} \\
& \cdot\left[\frac{1+\rho(1-2 p)}{2}\right]^{i-1-k}
\end{aligned}
$$

being $b_{i}\left(p_{0}, p\right)$ defined as

$$
b_{i}\left(p_{0}, p\right):=\left\lceil\frac{1}{2}\left(i-1+\frac{\log \left(\left(1-p_{0}\right) / p_{0}\right)}{\log ((1+\rho(1-2 p)) /(1-\rho(1-2 p)))}\right)\right\rceil
$$

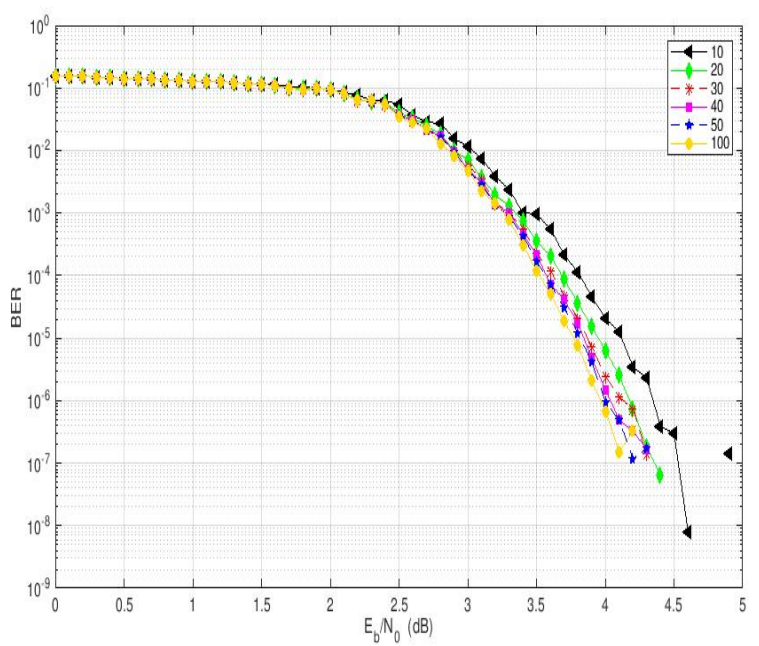

Fig. 1. BER vs. $E_{b} / N_{0}$ in $\mathrm{dB}$ of the rate-1/2 randomly chosen codes with distribution pairs $(\lambda, \rho)$ given in (13), called Code 22 in [4]. The performance curves have been obtained with an iteration number $I=10,20,30,40$, and 50 of the decoding algorithm.

Gallager's idea, resumed in [4], is then to find the supremum $p^{*}$ of all values of $p_{0}$ for which the sequence $p_{l}$ is monotonically decreasing and hence converges to 0 . He also proves that, as the block length of the code and girth of the graph grow large, this decoding algorithm works for all $p_{0}<p^{*}$.

\section{LOW COMPLEXITy DETERMINATION OF $p^{*}$}

Applying the method defined in [20], instead of searching the last value of the parameter $p_{0}$ granting the convergence of (7), we solve a problem of quadratic degeneracy which can be assigned to a standard software.

When the second partial derivative of $f\left(p_{0}, p\right)$ with respect to $p, f_{p p}\left(p_{0}, p\right)$, is $\neq 0$, the problem of quadratic degeneracy is the system of equations

$$
\left\{\begin{array}{l}
f\left(p_{0}, p\right)=p \\
f_{p}\left(p_{0}, p\right)=1
\end{array}\right.
$$

where $f_{p}\left(p_{0}, p\right)$ is the first partial derivative of $f\left(p_{0}, p\right)$ with respect to $p$.

The solution of (11) gives the value $p^{*}$, namely the maximum $p_{0}$ granting the convergence of (7).

\section{NuMERIC RESULTS}

With regard to irregular LDPC codes with $i_{1}>2$, as example we consider the rate-1/2 irregular LDPC codes given in [4] with $i_{1}=5$, having degree distributions:

$$
\begin{aligned}
\lambda(x)= & 0.496041 x^{4}+0.173862 x^{5}+0.077225 x^{20} \\
& +0.252871 x^{22} \\
\rho(x)= & x^{13}
\end{aligned}
$$

and

$$
\begin{aligned}
\lambda(x)= & 0.284961 x^{4}+0.124061 x^{5}+0.068844 x^{26} \\
& +0.109202 x^{28}+0.119796 x^{29}+0.293135 x^{99} \\
\rho(x)= & x^{21}
\end{aligned}
$$




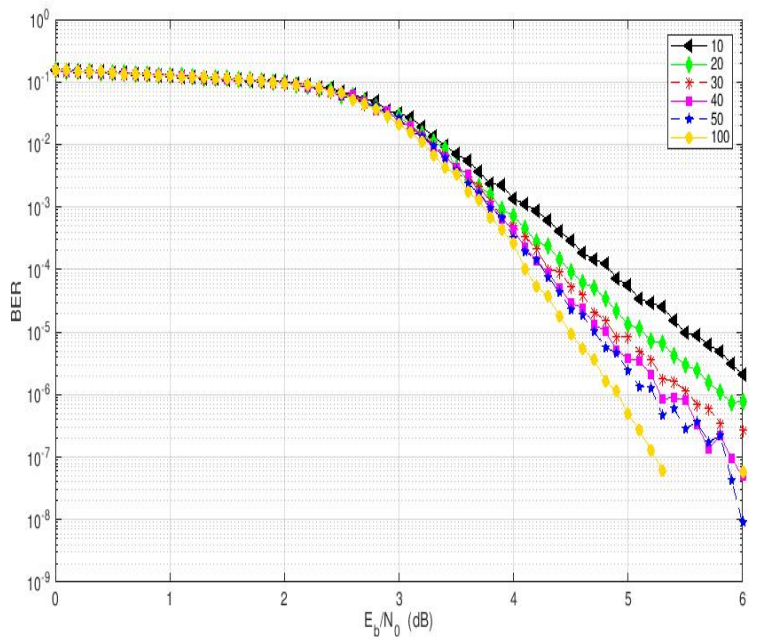

Fig. 2. BER vs. $E_{b} / N_{0}$ in $\mathrm{dB}$ of the rate-1/2 randomly chosen codes with distribution pairs $(\lambda, \rho)$ given in (12), called Code 14 in [4]. The performance curves have been obtained with an iteration number $I=10,20,30,40$, and 50 of the decoding algorithm.

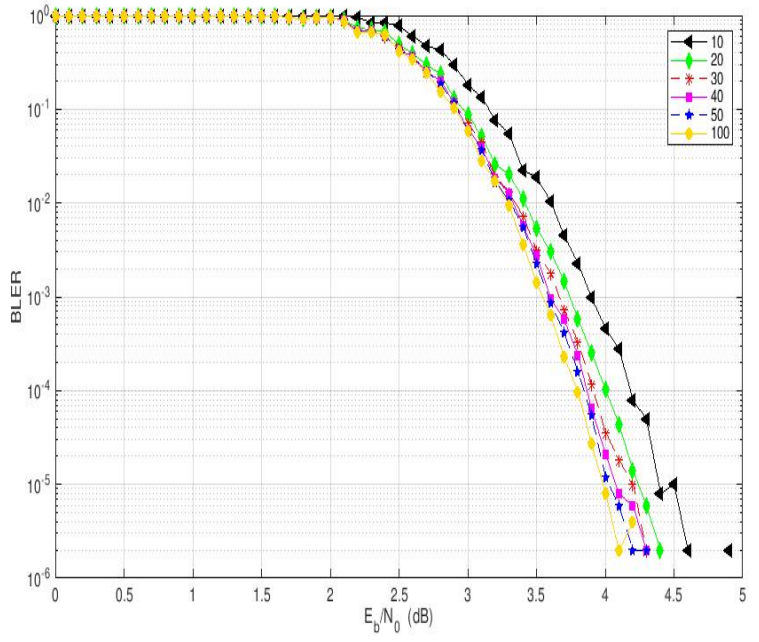

Fig. 3. BLER vs. $E_{b} / N_{0}$ in $\mathrm{dB}$ of the rate- $1 / 2$ randomly chosen codes with distribution pairs $(\lambda, \rho)$ given in (13), called Code 22 in [4]. The performance curves have been obtained with an iteration number $I=10,20,30,40$, and 50 of the decoding algorithm.

and with $i_{1}=3$, having degree distributions:

$$
\begin{aligned}
& \lambda(x)=0.123397 x^{2}+0.555093 x^{3}+0.321510 x^{15} \\
& \rho(x)=x^{9}
\end{aligned}
$$

and

$$
\begin{aligned}
\lambda(x)= & 0.093368 x^{2}+0.346966 x^{3}+0.159355 x^{20} \\
& +0.400312 x^{22} \\
\rho(x)= & x^{13}
\end{aligned}
$$

The first two codes (12) and (13), called Code 14 and Code 22 in [4], respectively, both present variable node degree distributions $\lambda(x)$ of minimum degree $i_{1}=5$ and, therefore,

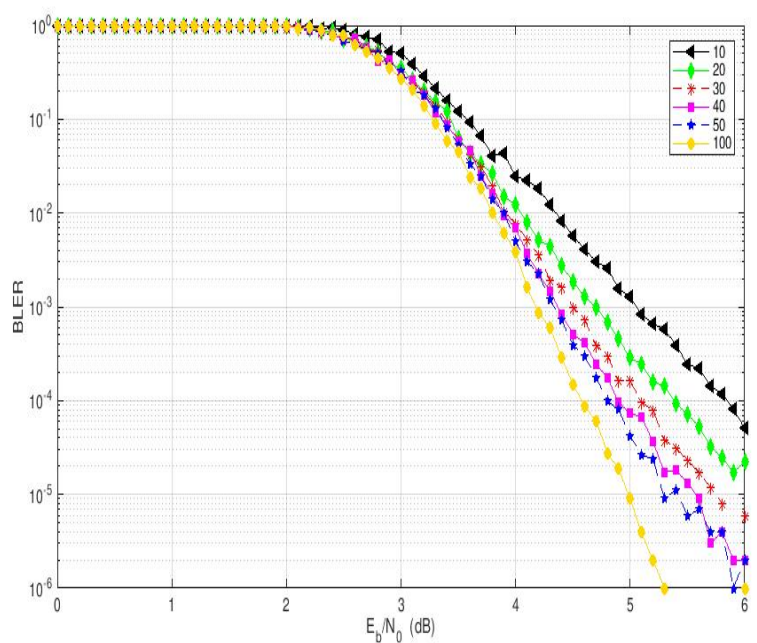

Fig. 4. BLER vs. $E_{b} / N_{0}$ in $\mathrm{dB}$ of the rate- $1 / 2$ randomly chosen codes with distribution pairs $(\lambda, \rho)$ given in (12), called Code 14 in [4]. The performance curves have been obtained with an iteration number $I=10,20,30,40$, and 50 of the decoding algorithm.

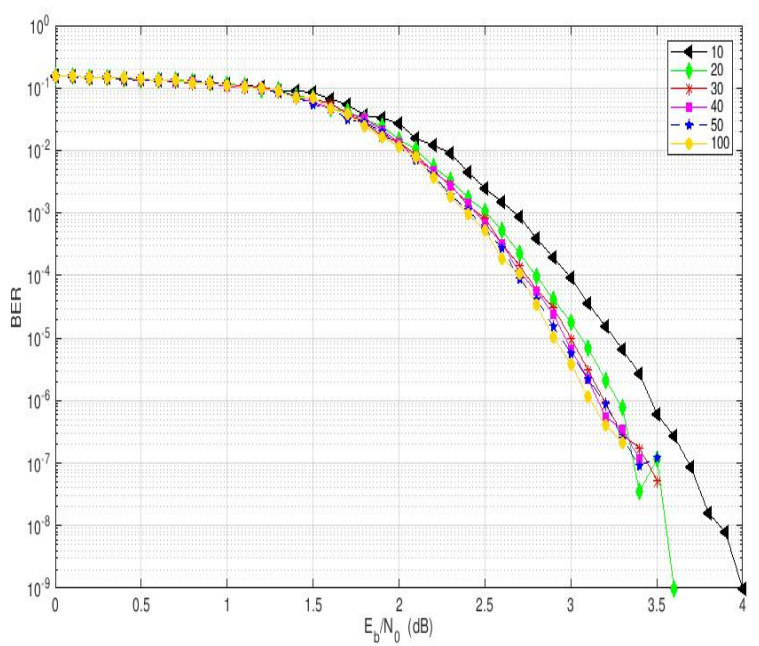

Fig. 5. BER vs. $E_{b} / N_{0}$ in $\mathrm{dB}$ of the rate-1/2 randomly chosen codes with distribution pairs $(\lambda, \rho)$ given in (15), called Code 14' in [4]. The performance curves have been obtained with an iteration number $I=10,20,30,40$, and 50 of the decoding algorithm.

this ensures that their graphs have good expansion, as proved in Lemma 3 of [4]. In fact, in this Lemma 3 it is shown that, when $i_{1} \geq 5$, the polynomial $\rho(x)$ has degree at most $J$ for some constant $J$. Secondly, it is demonstrated that, given the block length $n$, with probability $1-O(1 / n)$, for some fixed $\alpha>0, \epsilon>0$, and $\beta=3 / 4+\epsilon$, the bipartite graph of the code is an $(\alpha, \beta)$ expander. Thus, the analysis performed in [26] to determine the asymptotical behaviour of (18) in [15] for $t \rightarrow \infty$ can be applied (to find the upper bounds $\sigma_{\text {bound }}^{*}=\sqrt{\frac{2}{s_{\text {bound }}^{*}}}$, solution of (4)). Moreover, since, for the codes considered, $i_{1}>2$, the bounds $s_{\text {approx }}^{*}$ and $s_{\text {Jensen }}^{*}$, reported in [16], cannot be applied.

On the other hand, as far as the other two codes (14) and 


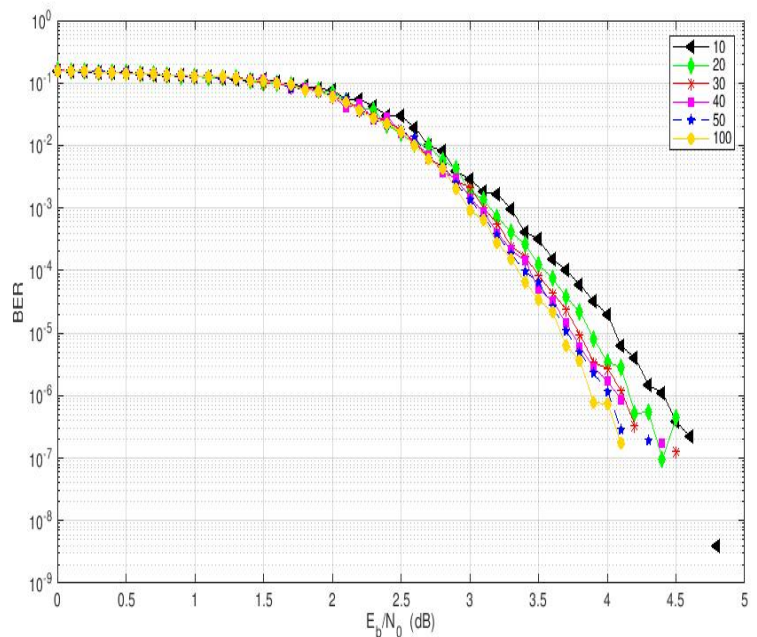

Fig. 6. BER vs. $E_{b} / N_{0}$ in $\mathrm{dB}$ of the rate-1/2 randomly chosen codes with distribution pairs $(\lambda, \rho)$ given in (14), called Code 10' in [4]. The performance curves have been obtained with an iteration number $I=10,20,30,40$, and 50 of the decoding algorithm.

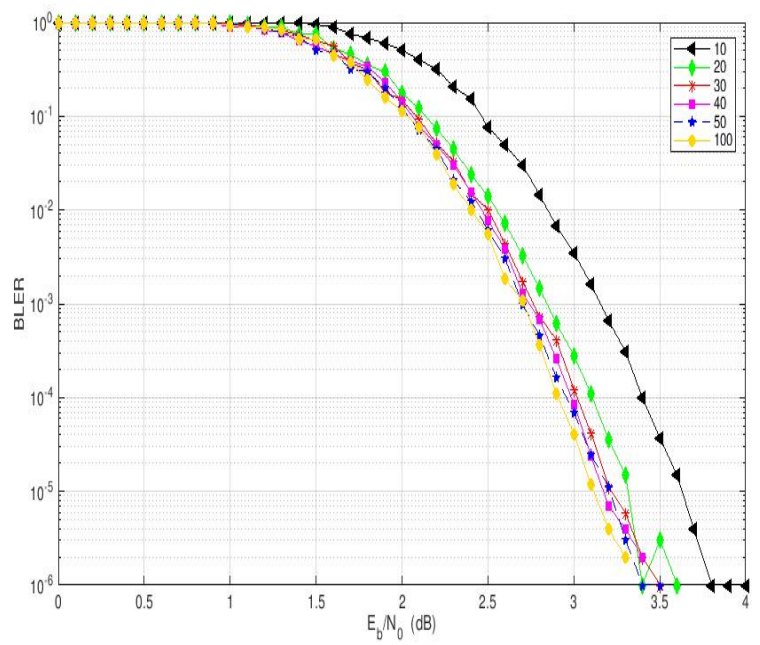

Fig. 7. BLER vs. $E_{b} / N_{0}$ in $\mathrm{dB}$ of the rate-1/2 randomly chosen codes with distribution pairs $(\lambda, \rho)$ given in (15), called Code 14' in [4]. The performance curves have been obtained with an iteration number $I=10,20,30,40$, and 50 of the decoding algorithm.

(15) are concerned, called Code 10' and Code 14' in [4], both present variable node degree distributions $\lambda(x)$ of minimum degree $i_{1}=3$ and have associated bipartite graphs that do not have a sufficient expansion (for Lemma 3 in [4] to hold). Thus, our above mentioned Lemma (1) cannot instead be applied.

The two codes with degree distribution polynomials (12) and (13), respectively, present a threshold upper bound $\sigma_{\text {bound }}^{*}=1.02239$ and $\sigma_{\text {bound }}^{*}=1.07602$, respectively. The Mathematica ${ }^{\circledR}$ script written to determine these results is reported in Appendix A of [15].

In [4], after defining $p_{l}$ as the probability that a variable node sends an incorrect message in round $l$ of a message passing decoding algorithm, the authors have determined, for each of the four rate-1/2 codes reported in Table I of [4],

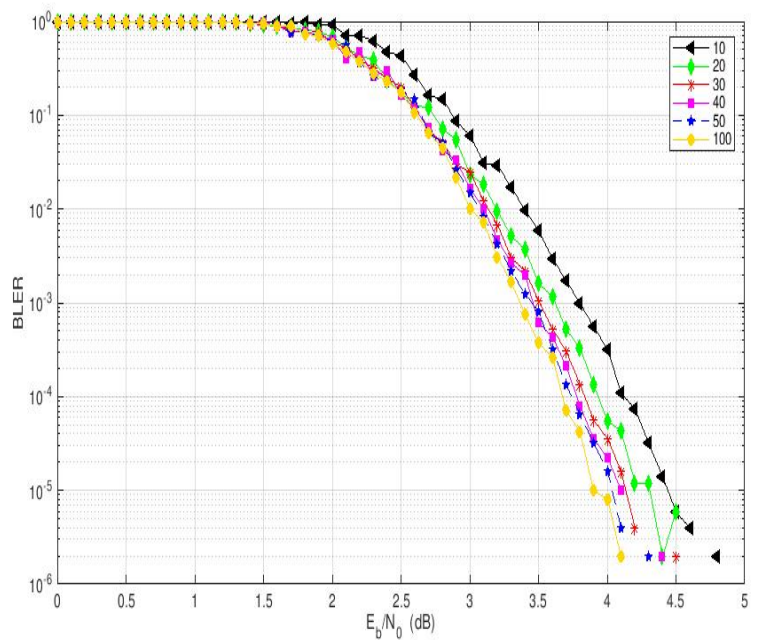

Fig. 8. BLER vs. $E_{b} / N_{0}$ in $\mathrm{dB}$ of the rate-1/2 randomly chosen codes with distribution pairs $(\lambda, \rho)$ given in (14), called Code 10' in [4]. The performance curves have been obtained with an iteration number $I=10,20,30,40$, and 50 of the decoding algorithm.

also the values $p^{*}$, defined as the "supremum of all values of $p_{0}$ for which the sequence $p_{l}$ is monotonically decreasing and hence converges to 0 ". The two codes with degree distribution polynomials (12) and (13), respectively, have $p^{*}$ values of 0.0505 and 0.0533 , respectively, when the transmission of coded symbols over a binary-symmetric channel is considered, whereas the two codes with degree distribution polynomials (14) and (15), respectively, have $p^{*}$ values of 0.0578 and 0.0627 , respectively.

Solving (11), instead, we found that the two codes with degree distribution polynomials (12) and (13), respectively, have $p^{*}$ values of 0.05053 and 0.05334 , respectively, whereas the two codes with degree distribution polynomials (14) and (15), respectively, have $p^{*}$ values of 0.05781 and 0.06272 , respectively, thus obtaining a perfect agreement with the results of [4].

As noticed in [4], " $p^{*}$ represents the error rate we would expect to be able to handle for arbitrarily long block lengths, and that we only expect to approach $p^{*}$ asymptotically in practice as the number of nodes grows". Thus, higher $p^{*}$ values lead to a better code performance, as shown in Figs. 3 and 4 of [4], where the experimental results with hard decision decoding are presented. As shown in Fig. 3 of [4], Code 22, presenting a $p^{*}$ value of 0.0533 , performs better than Code 14 , presenting a $p^{*}$ value of 0.0505 , and, similarly, as shown in Fig. 4 of the same paper, Code 14', presenting a $p^{*}$ value of 0.0627 , performs better than Code $10^{\prime}$, presenting a $p^{*}$ value of 0.0578 .

As expected, and as noted also in [18], where $\sigma^{*}$ and $p^{*}$ values have been compared in Tables I and II, the code characterized by a lower $p^{*}$ value, i.e., the code with degree distribution polynomials (12), also presents a lower threshold upper bound $\sigma_{\text {bound }}^{*}$ value. 


\section{A. Simulation Results}

Consider an ensemble of random codes with edgeperspective degree distributions $\lambda(x)$ and $\rho(x)$ given above. A custom software based on [14] (also used in [13] and [25] to design well performing rate compatible puncturing patterns for LDPC codes on the basis of the results of [16] and [20]) was employed to simulate their performance over an additive Gaussian noise channel (AWGN) channel, assuming a binary phase shift keying (BPSK) modulator. The belief propagation algorithm, also called message passing or sumproduct algorithm, commonly employed for LDPC decoding, has been adopted, employing soft decision.

In Figs. 1 and 2 are shown the bit error rate (BER) performance curves of some randomly chosen codes with distribution pairs $(\lambda, \rho)$ given in (12) and (13), called Code 14 and Code 22 in [4], respectively. As expected from the hard decoding experiments conducted in [4] and mentioned above, the BER performance obtained using Code 22 is better than that obtained with Code 14, since the first presents a higher $p^{*}$ value. As far as the block error rate performance (BLER) is concerned, reported in Figs. 3 and 4, the same conclusions still hold.

In Figs. 5 and 6 are shown the bit error rate (BER) performance curves of some randomly chosen codes with distribution pairs $(\lambda, \rho)$ given in (15) and (14), called Code 14' and Code 10' in [4], respectively. As expected from the hard decoding experiments conducted in [4] and mentioned above, the BER performance obtained using Code 14' is better than that obtained with Code 10', since the first presents a higher $p^{*}$ value. As far as the block error rate performance (BLER) is concerned, reported in Figs. 7 and 8, the same conclusions still hold.

\section{CONCLUSIONS}

Owing to their good performance, LDPC codes are an important family of error-correction codes employed in current data communication systems. In this paper, the analysis of irregular LDPC codes based on some low complexity upper bounds on their belief-propagation decoding thresholds, recently presented in [16], was addressed. This was possible thanks to the work in [17], upon which the derivation is based, and to the algorithmic method for LDPC codes threshold evaluation proposed in [20]. The results found in the paper show that the first low complexity upper bound on beliefpropagation decoding thresholds, published in [16], is useful for the class of irregular low-density parity-check (LDPC) codes for which it was conceived, i.e., for the LDPC codes characterized by variable node degree distributions $\lambda(x)$ of minimum degree $i_{1}>2$. However its validity is restricted to bipartite graphs that present a sufficient expansion, namely to the bipartite graphs of LDPC codes fulfilling the conditions given in Lemma 3 of [4]. The analysis published in [16] cannot be applied, instead, to bipartite graphs that do not have a sufficient expansion. Furthermore, we investigated the suitability of the algorithmic method presented in [20] also to carry out the performance analysis of the class of irregular near capacity achieving LDPC codes considered. The $p^{*}$ values, representing the error rate we would expect to be able to handle for arbitrarily long block lengths, obtained applying the quadratic degeneracy theory, have been shown to be in perfect agreement with those reported in [4]. Moreover, the simulation results obtained applying the belief propagation algorithm, employing soft decision, have been shown to be in perfect agreement with the hard decoding experimental results reported in the same paper.

\section{REFERENCES}

[1] R. G. Gallager, "Low-density parity-check codes", IRE Transactions on Information Theory, vol. 8, no. 1, January 1962, pp. 21-28.

[2] R. M. Tanner, "A recursive approach to low complexity codes", IEEE Transactions on Information Theory, vol. 27, no. 5, September 1981, pp. 533-547.

[3] David J. C. Mackay, "Good error correcting codes based on very sparse matrices", IEEE Transactions on Information Theory, vol. 45, no. 2, March 1999, pp. 399-431.

[4] M. G. Luby, M. Mitzenmacher, M. A. Shokrollahi, and D. A. Spielman, "Improved low-density parity-check codes using irregular graphs", IEEE Transactions on Information Theory, vol. 47, no. 2, February 2001, pp. 585-598.

[5] F. Babich, M. Comisso, and A. Dorni, "A Novel SIR-Based Access Scheme for Multi-Packet Communication in 802.11 Networks", Proceedings of the 2012 IEEE International Conference on Communications, ICC'12, Ottawa, Canada, June 10-15, 2012.

[6] R. Corrado, M. Comisso, and F. Babich, "On the impact of the video quality assessment in 802.11e ad-hoc networks using adaptive retransmissions", Proceedings of the IEEE IFIP Annual Mediterranean Ad Hoc Networking Workshop (Med-Hoc-Net), Piran, Slovenia, June 24, 2014, pp. 47-54.

[7] V. Sidorenko, M. Bossert, and F. Vatta, "Properties and encoding aspects of direct product convolutional codes", Proc. of the 2012 IEEE International Symposium on Information Theory, ISIT'12, Boston, U.S.A., July 1-6, 2012, pp. 2351-2355.

[8] B. Liu, Y. Li, B. Rong, L. Gui, and Y. Wu, "LDPC-RS Product Codes for Digital Terrestrial Broadcasting Transmission System", IEEE Trans. on Broadcasting, vol. 60, no. 1, March 2014, pp. 38-49.

[9] M. Comisso and F. Babich, "Coverage analysis for 2D/3D millimeter wave peer-to-peer networks", IEEE Transactions on Wireless Communications, vol. 18, no. 7, July 2019, pp. 3613-3627.

[10] F. Babich and M. Comisso, "Impact of segmentation and capture on slotted Aloha systems exploiting interference cancellation", IEEE Trans. on Vehicular Technology, vol. 68, no. 3, March 2019, pp. 2878-2892.

[11] F. Babich and M. Comisso, "Multi-packet communication in heterogeneous wireless networks adopting spatial reuse: capture analysis", IEEE Transactions on Wireless Communications, vol. 12, no. 10, October 2013, pp. 5346-5359.

[12] F. Babich, M. Noschese. A. Soranzo, and F. Vatta, "Low complexity rate compatible puncturing patterns design for LDPC codes", Journal of Communication Software and Systems (JCOMSS), vol. 14, no. 4, December 2018, pp. 350-358.

[13] F. Babich, M. Noschese, and F. Vatta, "Analysis and design of rate compatible LDPC codes", Proceedings of the 27th IEEE International Symposium on Personal, Indoor and Mobile Radio Communications, PIMRC '16, Valencia, Spain, September 4-8, 2016, pp. 1-6.

[14] A. Boscolo, F. Vatta, F. Armani, E. Viviani, and D. Salvalaggio, "Physical AWGN channel emulator for bit error rate test of digital baseband communication", Applied Mechanics and Materials, vols. 241244, 2013, pp. 2491-2495.

[15] F. Vatta, A. Soranzo, M. Comisso, G. Buttazzoni, and F. Babich, "Low complexity bounds on a class of irregular LDPC belief-propagation decoding thresholds", Proceedings of the International Conference on Software, Telecommunications and Computer Networks, SOFTCOM '20, Hvar, Croatia, September 17-19, 2020.

[16] F. Vatta, A. Soranzo, and F. Babich, "Low-complexity bound on irregular LDPC belief-propagation decoding thresholds using a Gaussian approximation", Electronics Letters, vol. 54, no. 17, August 2018, pp. 1038-1040.

[17] S.-Y. Chung, T. J. Richardson, and R. Urbanke, "Analysis of sumproduct decoding of low-density parity-check codes using a Gaussian approximation", IEEE Transactions on Information Theory, vol. 47, no. 2, February 2001, pp. 657-670. 
[18] T. J. Richardson, A. Shokrollahi, and R. Urbanke, "Design of capacityapproaching irregular low-density parity-check codes", IEEE Transactions on Information Theory, vol. 47, no. 2, February 2001, pp. 619-637.

[19] F. Vatta, A. Soranzo, M. Comisso, G. Buttazzoni, and F. Babich, "Performance study of a class of irregular LDPC codes through low complexity bounds on their belief-propagation decoding thresholds", Proceedings of the AEIT 2019 International Annual Conference, Florence, Italy, September 18-20, 2019.

[20] F. Babich, A. Soranzo, and F. Vatta, "Useful mathematical tools for capacity approaching codes design", IEEE Communications Letters, vol. 21, no. 9, September 2017, pp. 1949-1952.

[21] F. Vatta, F. Babich, F. Ellero, M. Noschese, G. Buttazzoni, and M. Comisso, "Performance study of a class of irregular LDPC codes based on their weight distribution analysis", Proc. of the 2019 International Conference on Software, Telecommunications and Computer Networks, Split, Croatia, September 19-21, 2019, pp. 1-6.

[22] F. Vatta, F. Babich, F. Ellero, M. Noschese, G. Buttazzoni, and M. Comisso, "Role of the product $\lambda^{\prime}(0) \rho^{\prime}(1)$ in determining ldpc code performance", Electronics (Switzerland), vol. 8, no. 12, December 2019, pp. 1-14.

[23] C. Di, T. J. Richardson, and R. Urbanke, "Weight distribution of lowdensity parity-check codes", IEEE Transactions on Information Theory, vol. 52, no. 11, Nov. 2006, pp. 4839-4855.

[24] H. Jin, A. Khandekar, and R. McEliece, "Irregular repeat-accumulate codes", Proceedings of the 2nd International Symposium on Turbo Codes \& Related Topics, Brest, France, September 4-7, 2000, pp. 18.

[25] F. Babich, M. Noschese, A. Soranzo, and F. Vatta, "Low complexity rate compatible puncturing patterns design for LDPC codes", Proceedings of the 2017 International Conference on Software, Telecommunications and Computer Networks, SoftCOM'17, Split, Croatia, September 21-23, 2017.

[26] F. Vatta, A. Soranzo, and F. Babich, "More accurate analysis of sumproduct decoding of LDPC codes using a Gaussian approximation", IEEE Communications Letters, vol. 23, no. 2, February 2019, pp. 230233.

[27] F. Vatta, A. Soranzo, M. Comisso, G. Buttazzoni, and F. Babich, "New explicitly invertible approximation of the function involved in LDPC codes density evolution analysis using a Gaussian approximation", Electronics Letters, vol. 55, no. 22, October 2019, pp. 1183-1186.

[28] S.-Y. Chung, On the construction of some capacity-approaching coding schemes. PhD Dissertation, Mass. Inst. Technol., Cambridge, MA, 2000. Available at http://dspace.mit.edu/handle/1721.1/8981

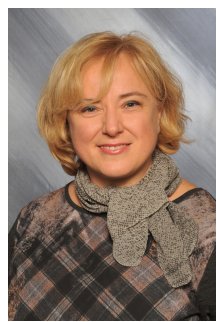

Francesca Vatta (M'98) received the M.Sc. Degree in Electronic Engineering and the Ph.D. Degree in Telecommunications from the University of Trieste, Trieste, Italy, in 1992 and 1998, respectively. She joined the Department of Engineering and Architecture (DIA) of the University of Trieste in 1999 where she is Assistant Professor of Information Theory and Error-Control Coding. Starting in 2002, she spent several months as visiting scholar at the University of Notre Dame, Notre Dame, IN, U.S.A., cooperating with the Coding Theory Research Group under the guidance of Prof. D. J. Costello, Jr. Starting in 2005, she spent several months as visiting scholar at the University of Ulm, Germany, cooperating with the Telecommunications and Applied Information Theory Research Group under the guidance of Prof. M. Bossert. She is an author of more than 100 papers published on international journals and conference proceedings. Her current research interests are in the area of channel coding concerning, in particular, the analysis and design of capacity achieving coding schemes.

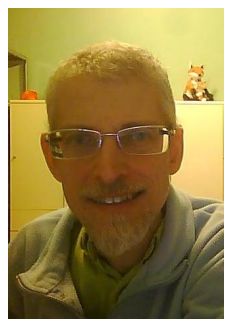

Alessandro Soranzo received the M.S. Degree in Mathematics from the University of Trieste, Trieste, Italy, in 1991. He joined the Department of Mathematics and Geosciences (DMG) of the University of Trieste in 1994, where he is Assistant Professor of Mathematical Analysis. He is an author of about 20 papers, published on international journals and conference proceedings, in the fields of convex tomography, topology of natural numbers, fluidodynamics, and the approximation of special function of statistics. His current research interests are in the area of the mathematical aspects of coding theory, mostly concerning capacity achieving coding schemes

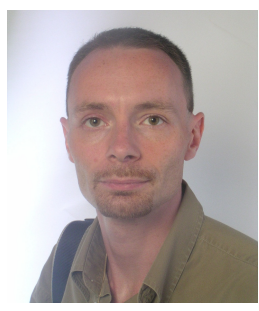

Massimiliano Comisso (M'09) received the Laurea degree in Electronic Engineering and the Ph.D. degree in Information Engineering from the University of Trieste, Italy. He was with Alcatel, where he worked on DWDM communication systems, and collaborated with Danieli Automation on electromagnetic NDE techniques. Currently, Massimiliano Comisso is an Assistant Professor at the Department of Engineering and Architecture (DIA) of the University of Trieste. He is author/co-author of more than 80 international scientific papers, and serves as referee/TPC member for several IEEE journals and conferences. He has been Best Student Paper Award (BPA) Finalist at GLOBECOM 2006 and received the BPA at CAMAD 2009. His research interests involve distributed wireless networks, millimeter-wave communications, antenna array synthesis, and small antennas.

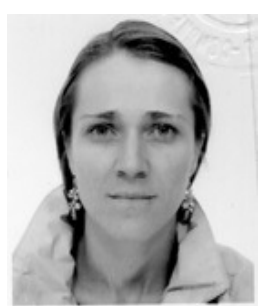

Giulia Buttazzoni received the Laurea degree (summa cum laude) in Telecommunication Engineering and the Ph.D. degree in Information Engineering from the University of Trieste (Italy), in 2008 and 2013, respectively. She is currently a researcher at the Department of Engineering and Architecture of the University of Trieste. Her research interests involve antenna array synthesis and numerical methods for electromagnetic fields.

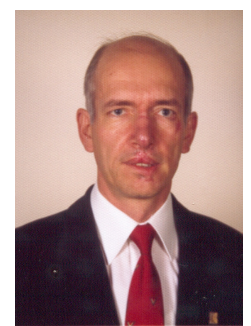

Fulvio Babich (SM'02) received the doctoral degree, (Laurea), cum laude, in Electrical Engineering, at the University of Trieste, on July 1984. After graduation he was with Telettra at the Research and Development Laboratories, where he was engaged in optical fiber communications. Then he joined Zeltron, where he was a communication system engineer, responsible of the activities within the ESPRIT program. In 1992 he joined the Department of Electrical and Electronic Engineering (DEEI) of the University of Trieste, where he is Professor of Digital Communications and Wireless Networks. Currently, Fulvio Babich is vice-director of the Department of Engineering and Architecture (DIA) and coordinator of the Ph.D. program for Industrial and Information Engineering of the University of Trieste. He is also member of the board of the National Telecommunications and Information Theory Group (GTTI), and was member of the Directive Board of the National Inter-University Consortium for Telecommunications (CNIT). His current research interests are in the field of wireless networks and millimeter-wave communications, where he is involved in channel modeling, multiple access techniques, channel encoding, error control techniques, and cross-layer design. 